Proceedings of the XXX GIRI Meeting; 2016 Sep 09 - 11; Netherland.

International Journal of High Dilution Research 2016; 15(4):32-35

Available online at www.highdilution.org

Conference presentation

\title{
Study of the action mechanisms of Arnica montana effects on macrophages
}

\author{
Marta Marzotto, Clara Bonafini, Debora Olioso, Paolo Bellavite \\ Department of Medicine, University of Verona, Strada Le Grazie 8, 37134 Verona, Italy
}

\begin{abstract}
Objective: To test the effect of Arnica montana (Arnica m.) on human macrophages.

Method: The human monocytic leukaemia cell line THP-1 was cultured and differentiated in mature macrophages with PMA and other differentiating agents. Macrophages were exposed to Arnica m. homeopathic dilutions (2c, 3c, 5c, 9c and 15c) or Control solvent. Total RNA was isolated and sequenced to perform quantitative evaluation.
\end{abstract}

Results: Screening sorted out with a list of 20 genes that were significantly changed by Arnica m. 2c treatment: 7 up-regulated and 13 down-regulated. Most notably, a clearly up-regulated function concerned the proteinaceous extracellutar matrix (ECM), including genes HSPG2, FBN2, FN1 $(p<0.01)$. The down-regulated genes converge into the common pathway of oxidative phosphorylation and particularly into the cell component gene ontology of mitochondrial complex I $(\mathrm{p}<0.001)$. Higher Arnica m. dilutions were effective also by upregulating the same geneset in cells treated with LPS but not in resting cells.

Conclusion: In synthesis, we have identified a group of genes that are upregulated by Arnica m. in macrophages and many of them are involved in the processes of connective tissue remodelling and regeneration.

\section{Background}

Arnica montana L. (referred to hele ss pathological conditions, including pain, stiffness and swelling associated with trauma and postoperative clinical conditions. There is some experimental evidence of an anti-inflammatory action in laboratory animals and in in vitro models (macrophages and fibroblasts) but its action deserve further investigation. Given the central role of macrophages in tissue repair and regeneration, we decided to evaluate this plant's effects in vitro on the THP-1 human cell line differentiated to macrophages with PMA, a widely used model for immune modulation. In a first study, we used RT-PCR analysis to investigate changes in the expression of a panel of 28 genes focused on immune response (1). In a second series of experiments we decided to re-investigate the same cell extracts with the most high-throughput method, RNA-sequencing (RNA-seq), designed to evaluate the whole transcriptome.

\section{Materials and Methods}

The human monocytic leukaemia cell line THP-1 was purchased from DSMZ (Germany). Arnica $m$. was produced by Boiron Laboratoires (Lyon, France) according to the French Homeopathic pharmacopoeia and provided as a first centesimal dilution (Arnica m. 1c) of the hydroalcoholic extract (Mother Tincture, MT) in 30\% ethanol/distilled water. Starting from a 1c solution, serial

Cite as: Marzotto M, Bonafini C, Olioso D, Bellavite P. Proceedings of the XXX GIRI Meeting; 2016 Sep 0911; Netherland. Int J High Dilution Res. 2016;15(4):32-35 
Proceedings of the XXX GIRI Meeting; 2016 Sep 09 - 11; Netherland. International Journal of High Dilution Research 2016; 15(4):32-35

Available online at www.highdilution.org

100x dilutions were prepared in $5 \mathrm{ml}$ of $30 \%$ ethanol/distilled water solvent followed by filtering and succussion. The last centesimal dilution step was always performed immediately before each experiment, in ultra-pure water. The THP-1 cell line was cultured in RPMI 1640 medium, supplemented with FBS $10 \%$ and $2 \mathrm{mM}$ final concentration of Ultraglutamine (Lonza), at $37^{\circ} \mathrm{C}$ in $5 \% \mathrm{CO} 2$ in a humidified incubator as described. On day 2 all the cell cultures were supplemented with $20 \mathrm{ng} / \mathrm{mL}$ of PMA and on day 3 the cultures were treated with IL- 4 at a concentration of $50 \mathrm{ng} / \mathrm{mL}$ for $24 \mathrm{~h}$ to differentiate them in "wound-healing" macrophages (M2type). In separate experiments we also cultivated PMA-differentiate THP-1 cell in the absence of further stimulants ("Resting") or in the presence of LPS at a concentration of $10 \mathrm{ng} / \mathrm{mL}$ ("Lowdose LPS"). Macrophages were exposed to Arnica m. dilutions or Control solvent ( $1 \mathrm{ml}$ cell culture $+110 \mu$ l test solutions). Cell viability was checked by the Cell proliferation reagent WST1 assay. Total RNA from cultured THP-1 cells was isolated using the RNeasy mini Kit (Qiagen). RNA aliquots were used to isolate poly(A) mRNA for the preparation of a directional Illumina RNA-Seq library using the TruSeq RNA Sample Prep Kit v2 (Illumina Inc., San Diego, CA, USA). Libraries were evaluated by RT-PCR and sequenced with a NextSeq500 sequencer. Genes with Log2 Fold Change values that were significantly positive (up-regulated) or negative (downregulated) were defined as differentially expressed genes (DEGs). RNA-seq was done on 5 separate experiments in IL4-cells treated with Arnica m. 2c dilution to identify DEGs with high statistical significance according to FDR scoring (2). We then tested pooled samples of the experiments carried out with $2 \mathrm{c}$ and higher dilutions, namely $3 c, 5 c, 9 c$ and $15 c$. The statistical significance of the differences between expression profiles of gene groups (up-regulated) from cells treated with various Arnica m. dilutions (2c, 3c, 5c, 9c, 15c) was calculated by the Friedman multi-sample test followed by the Wilcoxon signed-rank test using the SPSS software, version 17 (SPSS Inc., Chicago, IL, USA).

\section{Results}

The WST assay of cell viability showed that the metabolicactivity of macrophages, both in the resting state and after IL-4 differentiation, was slightly increased after $24 \mathrm{~h}$ incubation with Arnica $\mathrm{m}$. dilutions $2 \mathrm{c}$ and $3 \mathrm{c}$, but the difference with the Control vehicle was not statistically significant.

The RNA-seq analysis performed on IL-4 differentiated cells treated with Arnica m. 2c (final concentration of 10-8 sesquiterpene lactones) sorted out a list of 20 genes which expression was significantly changed (2): 7 up-regulated and 13 down-regulated. The 7 up-regulated genes included low-density lipoprotein-receptor-related protein 1 (LRP1), fibronectin 1 (FN1), lysine (K)-specific methyltransferase (KMT2D), complement component receptor 1 (CR1), heparan sulfate proteoglycan (perlecan, HSPG2), microtubule-actin crosslinking factor 1(MACF1), and fibrillin 2 (FBN2). The set of down-regulated DEGs (13 genes) were mitochondrial genes coding for proteins of the mitochondrial respiratory chain complex.

Functional gene enrichment analysis showed that among the genes stimulated by Arnica m., a statistically significant enrichment of genes with EGF-like (LRP1, HSPG2, FBN2, FN1) and Calcium ion binding motifs (LRP1, MACF1, FBN2) emerged ( $\mathrm{p}<0.001$ and $<0.05$ respectively). Most notably, a clearly up-regulated function concerned the proteinaceous extracellular matrix (ECM), including genes HSPG2, FBN2, FN1 ( $\mathrm{p}<0.01)$. Fibronectin was identified in considerable amounts in cell culture supernatants and was increased by Arnica m. 2c treatment (2). The down-regulated genes converge into the common pathway of oxidative phosphorylation and particularly into the cell component gene ontology of mitochondrial complex I $(p<0.001)$. However, the decrease of mitochondrial genes did not impair cell viability.

Cite as: Marzotto M, Bonafini C, Olioso D, Bellavite P. Proceedings of the XXX GIRI Meeting; 2016 Sep 0911; Netherland. Int J High Dilution Res. 2016;15(4):32-35 
We investigated the up-regulating changes induced by increasingly higher Arnica $\mathrm{m}$. dilutions (Figure 1).
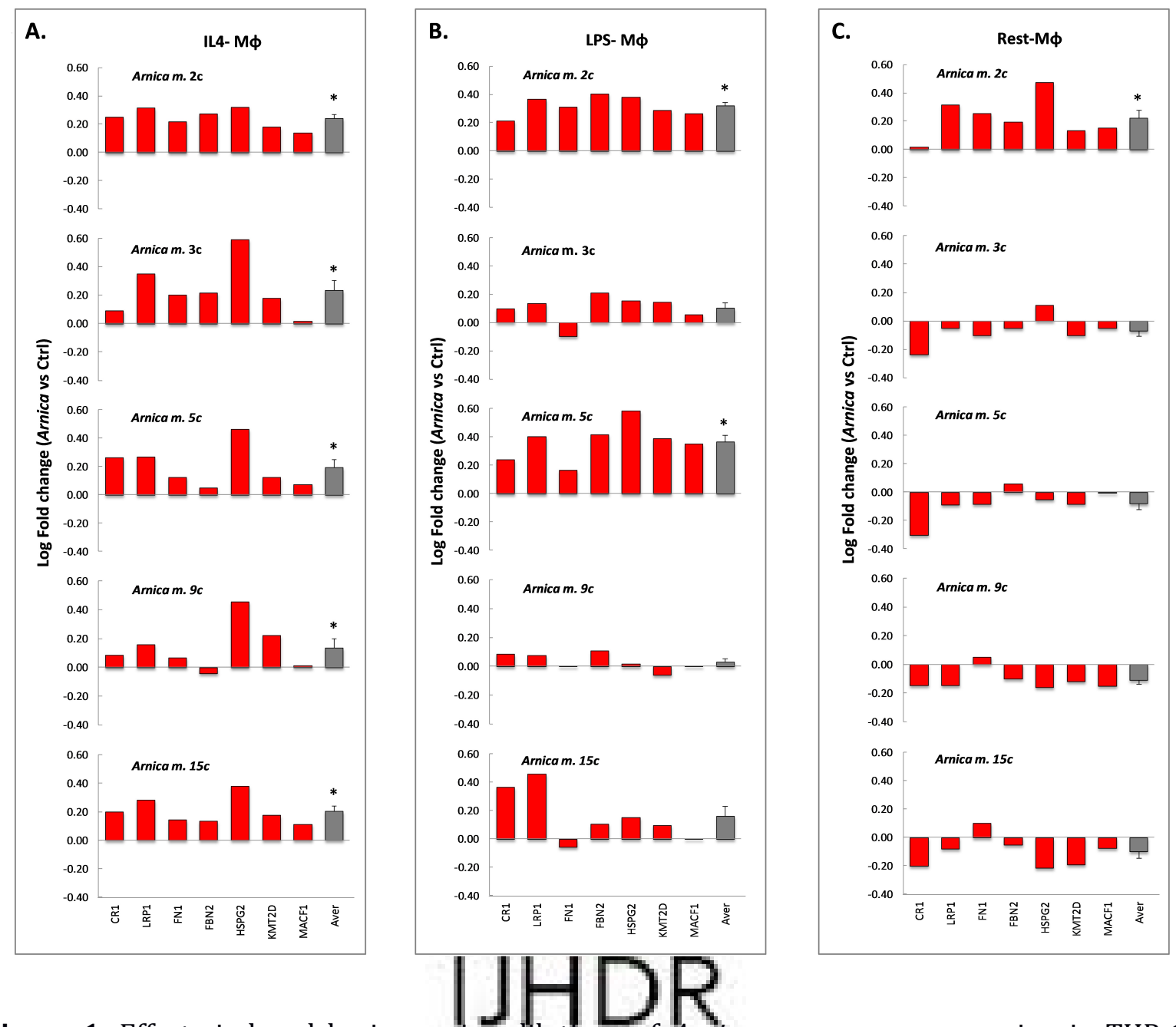

Figure 1. Effects induced by increasing dilutions of Arnica $m$. on gene expression in THP-1 macrophages $(M \phi)$. Red bars report log fold changes of single genes, grey bars report the mean fold changes \pm SE of the whole group considered as geneset at each dilution tested. Panel A: IL4differentiated cells. Panel B: LPS-activated cells. Panel C: Resting cells. ${ }^{*}$ Wilcoxon p values $<0.05$.

These studies were carried out in pooled samples of RNA extracted from 5 separate experiments. IL-4 treated macrophages (Figure 1, panel A) showed an increase of the indicated genes at all dilutions, suggesting that these cells are extremely sensitive to the effect of the medicinal plant. Arnica m. 3c similarly showed a prevailing stimulating effect in the previously up-regulated geneset: 6 genes out of a total of 7 were here also be found up-regulated. The average changes of each geneset (grey bars) were approximately of the same magnitude as those induced by Arnica m. 2c and were statistically significant. Arnica m. 5c, 9c and 15c stimulated all genes of the up-regulated geneset in a statistically significant way, although with a slightly lower effect size. In summary, up-regulating effects were maintained across the increasing dilutions. Interestingly, LPS-treated cells (Figure 1, panel B) were sensitive to this remedy at $2 \mathrm{c}$ as IL4-differentiated macrophages, but also at the higher dilution 5c. Paradoxically, the effect decreased from $2 \mathrm{c}$ to $3 \mathrm{c}$ as expected according to a conventional pharmacological 
Proceedings of the XXX GIRI Meeting; 2016 Sep 09 - 11; Netherland. International Journal of High Dilution Research 2016; 15(4):32-35

Available online at www. highdilution.org

wisdom, but, from 3c to 5c, the effect of Arnica m. increased again, even if the dilution between the two drug samples is 4 orders of magnitude (10,000 times). Resting cells (Figure 1, panel C) showed a up-regulating effect of the same gene group as observed in the other conditions, thus confirming the sensitivity of those genes to the stimulus by Arnica m.. However, in resting cells Arnica $m$. higher dilutions did not have significant effects and, on the contrary, a trend to

inhibition was evident, even if it did not reach the statistical threshold. Inspection of dose responses of individual genes and of the averages in all described conditions (Figure 1 panels A, $B, C)$ reveals a marked non-linearity: in several instances (but not in all the cases) alternating peaks of different activities with pseudo-sinusoidal trends are observed, typical of homeopathic paradoxical phenomena (3). Down-regulation did not give consistent results in different conditions: in resting cells and in cells treated with LPS, no or very little and variable downregulation of mitochondrial genes appeared (data not shown).

In synthesis, we have identified a group of genes that are upregulated by Arnica $\mathrm{m}$. in macrophages and many of them are involved in the processes of connective tissue remodelling and regeneration. The fact that the same group of genes undergo to the same trend of regulation in different conditions of cell cultivation reinforces the hypothesis that these are true "target" genes of this remedy. Furthermore, our data suggest that the state of cell differentiation is a fundamental factor in determining the sensitivity to ultra-low doses and high dilutions.

\section{Conclusions}

The results of this work indicate that homeopathic Arnica m. acts on macrophages by modulating a number of genes. RNA-seq analysis allowed the identification of several genes which are particularly sensitive to ultra-low doses and high dilutions of this plant extract. The main and novel finding is the increased expression of several genes of tissue matrix proteins and particularly of fibronectin. This evidepce indicatesomprevant targets of Arnica m. in woundhealing processes.

\section{Acknowledgments}

This work was supported by a scientific agreement between University of Verona, Dep. of Medicine, and Boiron Laboratoires (Lyon). The authors have no conflict of interest.

\section{References}

1. Olioso D, Marzotto M, Bonafini C, Brizzi M, Bellavite P. Arnica montana effects on gene expression in a human macrophage cell line. Evaluation by quantitative Real-Time PCR. Homeopathy. 2016; 105: 131-147.

2. Marzotto M, Bonafini C, Olioso D, Baruzzi A, Bettinetti L, Di Leva F, Galbiati E, Bellavite P. Arnica montana stimulates extracellular matrix gene expression in a macrophage cell line differentiated to wound healing phenotype. PloseONE. 2016; doi:10.1371/journal.pone.0166340 (in press).

3. Bellavite P, Signorini A, Marzotto M, Moratti E, Bonafini C, Olioso D. Cell sensitivity, nonlinearity and inverse effects. Homeopathy. 2015; 104: 139-160.

(C) International Journal of High Dilution Research.

Not for commercial purposes.

Cite as: Marzotto M, Bonafini C, Olioso D, Bellavite P. Proceedings of the XXX GIRI Meeting; 2016 Sep 0911; Netherland. Int J High Dilution Res. 2016;15(4):32-35 